\title{
Evaluation of the Effects Caused by Mandibular Advancement Devices Using a Numerical Simulation Model
}

\author{
Marco Mandolini ${ }^{1(凶)}$, Manila Caragiuli ${ }^{1}$, Daniele Landi ${ }^{4}$, Antonio Gracco ${ }^{2}$, \\ Giovanni Bruno ${ }^{2}$, Alberto De Stefani ${ }^{2}$, and Alida Mazzoli ${ }^{3}$ \\ 1 Department of Industrial Engineering and Mathematical Sciences, Università Politecnica delle \\ Marche, Via Brecce Bianche 12, 60131 Ancona, Italy \\ m.mandolini@univpm.it \\ 2 Department of Neurosciences, University of Padua, Via Giustiniani, 5, 35128 Padua, Italy \\ 3 Department of Materials, Environmental Sciences and Urban Planning, Università Politecnica \\ delle Marche, Via Brecce Bianche 12, 60131 Ancona, Italy \\ 4 Department of Management, Information and Production Engineering, \\ University of Bergamo, Via Pasubio 7/b, 24044 Dalmine, BG, Italy
}

\begin{abstract}
Obstructive sleep apnea syndrome (OSAS) is a sleep disorder that causes pauses in breathing or periods of shallow breathing during sleep. Mandibular advancement devices (MADs) represent a non-invasive treatment for OSAS that has had the highest development in recent years. Nevertheless, literature has not primarily investigated the effects of mandibular advancement. This paper presents a finite element method numerical simulation model for evaluating the stress/strain distribution on the temporomandibular joint (TMJ) and periodontal ligaments caused by advancement devices used for the treatment of OSAS. Results highlight that the mandible lift phase generates significant stress values on TMJ, which cannot be neglected for extended usage of MADs. Furthermore, mandible molar teeth are more loaded than incisor ones.
\end{abstract}

Keywords: Mandibular advancement device · Obstructive Sleep Apnea Syndrome $\cdot$ Finite element method · Virtual prototyping · Computer-Aided Design

\section{Introduction and Literature Review}

Repeated episodes of complete (apnea) or partial (hypopnea) obstruction of the upper airway during sleep characterize the obstructive sleep disorders. The obstructive sleep apnea syndrome (OSAS) may affect the general health of a large percentage of the population, with a defined prevalence of approximately $6 \%$ for men and $4 \%$ for women, with data increasing in the last few years [1].

Oral Appliances represents a non-invasive alternative treatment for OSAS that had the most considerable development in recent years, and medical literature has demonstrated their effectiveness in reducing the number of respiratory events and in decreasing

(C) The Author(s) 2021

L. Roucoules et al. (Eds.): JCM 2020, LNME, pp. 101-107, 2021.

https://doi.org/10.1007/978-3-030-70566-4_17 
symptoms [2]. Mandibular advancement devices (MAD) are nocturnal intraoral appliances that stabilize the upper airways and increase their diameters, reducing pharynx collapsibility. These devices must be used every night and necessarily have some shortterm side effects (e.g., sialorrhea or xerostomia, headache, temporomandibular joints discomfort, dental soreness, and occlusal changes upon awakening). In the long term, on the other hand, there may be overjet and overbite reduction and decrease in the quantity and intensity of posterior occlusal contacts.

In the past, the finite element method (FEM) was used mainly for simulating stress and strain distribution on the temporomandibular joint (TMJ). The masticatory system under normal occlusal loads [3], the mandibular protrusion and opening were the typical configurations analysed. FEM also permits to evaluate periodontal ligaments (PDL) effects on mandibular stiffness [4], on teeth movement [5], or in case of bone loss [6]. Concerning OSAS, Brunzini et al. [7] and Crivellin et al. [8] respectively presented a simulation model toward the study of the effects caused by different MADS and the strength distribution on TMJ using MADs. However, the presented virtual prototype is only a preliminary result that deserves further improvement.

This paper presents a numerical simulation model for studying the stress/strain distribution on TMJ and PDL caused by MADs used for treating OSAS. The simulation model was used to characterize a specific patient and related MAD. This research work that can be intended as a protocol is a step toward understanding and evaluating the side effects of MADs. Results achievable by employing the presented simulation model on a more significant number of patients and MADs will permit practitioners to gain knowledge for improving the current golden rules for prescribing such devices.

\section{Materials and Methods}

The development of the numerical simulation model consisted of a combination of (i) the 3D anatomical model of TMJ and PDL, (ii) the materials, and (iii) the boundary conditions (Fig. 1). The first step of the methodology consisted of gathering computerized tomographic (CT) images of an OSAS affected patient, anatomical atlas (specific for TMJ and PDL), a physical prototype of a MAD, and the plasters casts of the mandibular and maxillary dental arches of the same patient. Such information allowed authors to build the complete 3D geometry of the simulation model. The second step consisted in defining the boundary conditions of the system, strictly related to the mandible rototranslation caused by the MAD. The third step aimed at establishing the constitutive equations of the material to be assigned to the anatomical geometry of the model. After having meshed the model (fourth step), the simulation model was ready to be used.

\subsection{Anatomical Model}

The 3D geometry reconstruction of the anatomical model followed different techniques. Temporal bone, mandible, and teeth were modelled employing an image processing software for 3D design and modelling of geometries from CT images of the patient's skull. Soft tissues (articular disc, joint capsule, anterior ligament, retrodiscal tissue, sphenomandibular (SPL), and stilomandibular (STL) ligaments), which cannot be univocally 


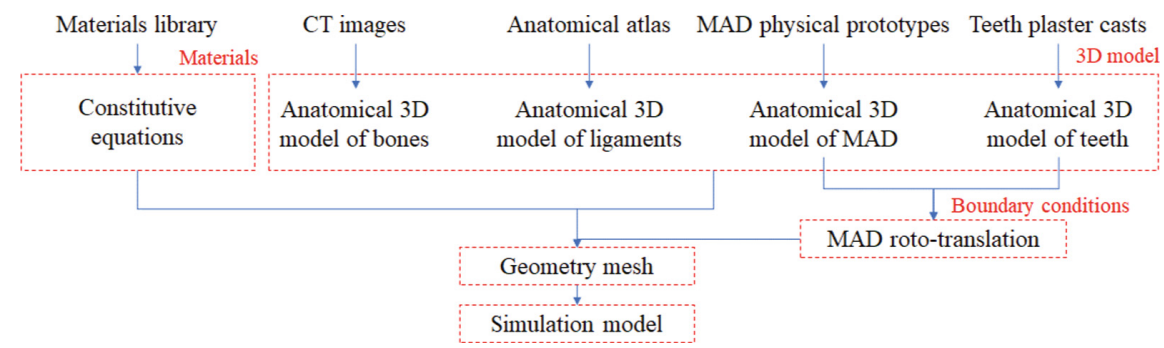

Fig. 1. The methodology used for developing the numerical simulation model.

determined from CT images, were modelled considering anatomical atlas and scientific papers (e.g., [9]). PDL ligaments were shaped by offsetting teeth roots of a constant value (i.e., $0.3 \mathrm{~mm}$ ). The mandibular MAD splint was modelled through a reverse engineering process because such devices are custom-made and handcrafted by practitioners, and the 3D CAD model is commonly unavailable. To be noted that, since the symmetry of a human skull, only half side of the geometry was modelled. Furthermore, the simulation model contains only the mandibular splint; the maxillary one is beyond the scope of this research.

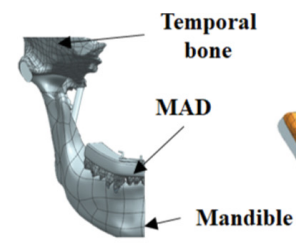

(a)

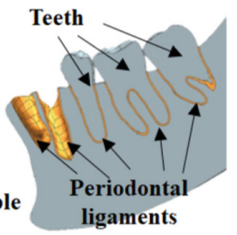

(b)

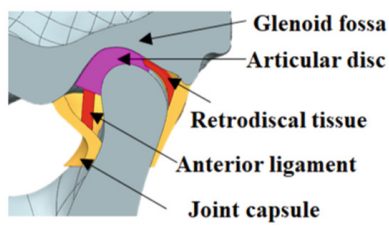

(c)

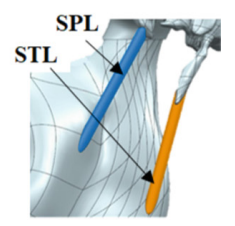

(d)

Fig. 2. Complete anatomical model. Frontal view of the 3D model (a), sagittal view of PDL (b), sagittal view TMJ (c), and sagittal view of SPL an STL (d).

\subsection{Materials}

Various materials were used for simulating the anatomical geometry. Materials models were retrieved from the literature. In particular, the articular disc, joint capsule, and all the TMJ ligaments were modelled using a Neo-Hookean material model [10]. In contrast, the $2^{\text {nd }}$ order Ogden model was adopted to model the PDLs [11]. The teeth and the mandible have been modelled as linear elastic, isotropic, and homogeneous [12]. MAD is a stratified element of three different materials. It consists of a thin inner layer in contact with the teeth and made of soft material (TPU), a middle between the inner and outer layers made of rigid material (PETG), and an outer layer of rigid material (PMMA). Such materials were modelled using a linear elastic model.

\subsection{Boundary Conditions}

The simulation model aims to evaluate the effects caused by the mandible roto-translation when inserting the MAD into the mouth (Fig. 3). First of all, it was necessary to assess 
the mandible translation, measured in "lift" and "advancement" according to a reference system based on the mandibular occlusal plane (defined when the mandible is resting). Lift is related to the mandible opening required when dressing the MAD. Advancement, instead, refers to the mandible protrusion caused by the MAD adjustment during the OSAS treatment (measured on the occlusal plane). Mandible rotation (Fig. 3c) is the angle between the occlusal plane of the mandible in the resting state and the same plane of the protruded jaw (Fig. 3b).

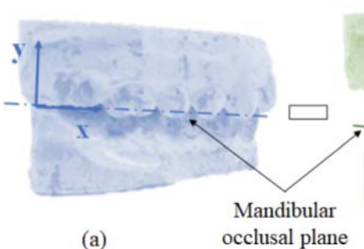

(a)

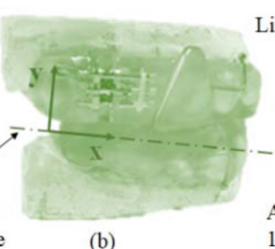

(b)

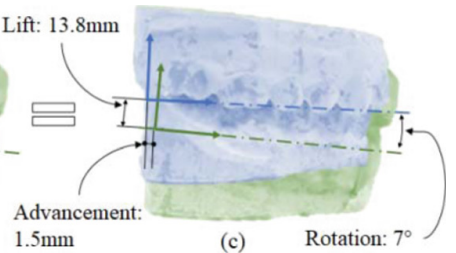

(c)

Fig. 3. Procedure for evaluating the mandible roto-translation. Teeth plaster casts in the rest position (a), teeth plaster casts combined with mandibular and maxillary MAD splints (b), and calculation of the mandible roto-translation, lift, advancement, and rotation (c).

Three types of joints were used for connecting anatomical elements: (i) bonded: no sliding or separation between surfaces is allowed, (ii) frictionless: sliding between surfaces without friction and (iii) frictional: surfaces can transmit shearing loads up to a specific value, before sliding. Bonded was used for almost all the ligaments apart between the articular disc and glenoid fossa (frictionless) and articular disc and joint capsule (frictional). Moreover, the following contacts were set between these interfaces: (i) $M A D$ and teeth, (ii) teeth and $P D L$, and (iii) $P D L$ and mandible. Regarding the analysis settings, an iterative solver was selected before running the simulation.

\subsection{Meshing}

The whole model consists of about 120000 10-nodes tetrahedral elements. The element size was adjusted for each geometry for an accurate meshing. The Skewness and the Orthogonal Quality mesh metrics criteria reported an average value $\mathrm{q}=0.29$ (standard deviation 0.19 ) and $\mathrm{q}=0.71$ (standard deviation 0.19 ), respectively, resulting in an excellent mesh quality.

\section{Results and Discussions}

The method presented in Fig. 1 was adopted for characterizing stress, strain, deformation and loads of a specific patient's TMJ, PDL, teeth, and MAD (Fig. 4a). The simulation model allowed authors to understand the deformation of the articular disc and joint capsule (Fig. 4b). For a high value of advancement, the articular disc clashes against the joint capsule, and this behaviour determines a higher stress value on the anterior area of the latter (Fig. 4c). SPL and STL ligaments are homogeneously stressed along their axes (Fig. 4d). While the most stressed and displaced teeth are the molars (Fig. 4e and 
Fig. 4f). To be noted that practitioners are mainly interested to the stress distribution rather than exact values: this is the reason why Fig. 4 does not contain a scale.

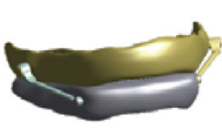

(a)

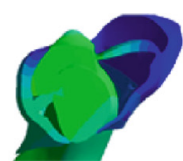

(b)

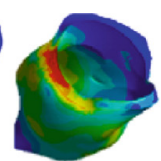

(c)

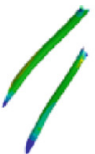

(d)

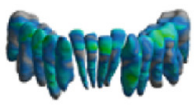

(e)

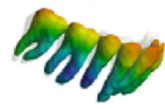

(f)

Fig. 4. MAD shape (a), TMJ deformation (b), TMJ stress (c), SPL and STL ligaments stress (d), teeth von-Mises stress (e) and teeth displacement (f).

Results also allowed to analyse the trend of TMJ stress (Fig. 5a) and SPL-STL force reaction (Fig. 5b) vs. The mandible roto-translation. It is worth noting that the TMJ stress gets the maximum stress approximately at $50 \%$ of roto-translation. Concerning the second set of simulation, mandible molar teeth are the most loaded, while mandible incisors are the least ones (Fig. 5c).

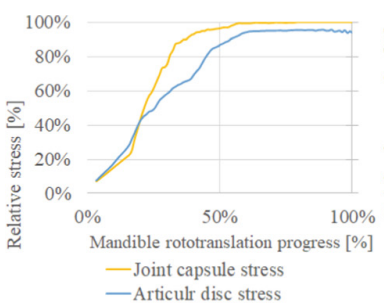

(a)

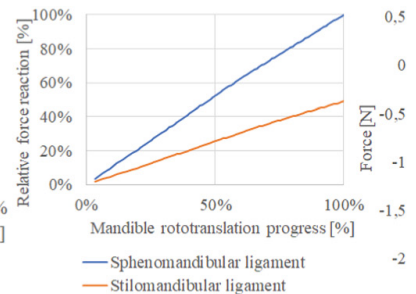

(b)

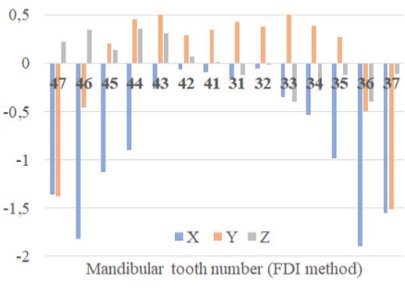

(c)

Fig. 5. TMJ stress (a) and SPL-STL force reaction (b) vs. Mandible roto-translation, PDL force reaction (c).

\section{Conclusions}

The paper presented a numerical simulation model for evaluating the effects caused by mandibular advancement devices, used for treating OSAS, on the temporomandibular joint and periodontal ligaments. The simulation model was used for characterising a specific patient and MAD (maxillary and mandibular splints connected through two symmetric metallic rods). The results highlight that the mandible lift phase generates significant stress values on TMJ, which cannot be neglected for prolonged usage of MADs. For this specific MAD, mandibular molars are more loaded than incisors.

For the feature, authors are going to analyse also the maxillary splint and arch. Furthermore, the authors will analyse the relationships among MADs (e.g., different materials, advancement system, geometry), patients (e.g., different apnea-hypopnea index - AHI), and the results presented in this paper (i.e., TMJ-PDL stress and teeth load/stress/displacement). By increasing the number of results, it will be possible to 
improve the current best-practices in prescribing MADs. Practitioners will be capable of advising MADs according to the patient's skeleton and AHI to minimize side-effects of such devices.

\section{References}

1. Chan, C.H., Wong, B.M., Tang, J.L., Ng, D.K.: Gender difference in snoring and how it changes with age: systematic review and meta-regression. Sleep Breath 16, 977-986 (2012). https://doi.org/10.1007/s11325-011-0596-8

2. Ramar, K., et al.: Clinical practice guideline for the treatment of obstructive sleep Apnea and snoring with oral appliance therapy: an update for 2015. J. Clin. Sleep Med. 11(7), 773-827 (2015). https://doi.org/10.5664/jcsm.4858

3. Citarella, R., Armetani, E., Caputo, F., Naddeo, A.: FEM and BEM analysis of a human mandible with added temporomandibular joints. The Open Mech. Eng. J. 6, 100-114 (2012). https://doi.org/10.2174/1874155X01206010100

4. Groning, F., Fagan, M.J., O'Higgins, P.: The effects of the periodontal ligament on mandibular stiffness: a study combining finite element analysis and geometric morphometrics. J. Biomech. 44(7), 1304-1312 (2011). https://doi.org/10.1016/j.jbiomech.2011.01.008

5. Qian, H., Chen, J., Katona, T.R.: The influence of PDL principal fibers in a 3-dimensional analysis of orthodontic tooth movement. Am. J. Orthod. Dentofac. Orthop. 120(3), 272-279 (2001). https://doi.org/10.1067/mod.2001.116085

6. Jeon, P.D., Turley, P.K., Ting, K.: Three-dimensional finite element analysis of stress in the periodontal ligament of the maxillary first molar with simulated bone loss. Am. J. Orthod. Dentofac. Orthop. 119(5), 498-504 (2001). https://doi.org/10.1067/mod.2001.112999

7. Brunzini, A., Gracco, A., Mazzoli, A., Mandolini, M., Manieri, S., Germani, M.: Preliminary simulation model toward the study of the effects caused by different mandibular advancement devices in OSAS treatment. Comput. Methods Biomech. Biomed. Engin. 21(13), 693-702 (2018). https://doi.org/10.1080/10255842.2018.1511776

8. Crivellin, G., Bruno, G., De Stefani, A., Mazzoli, A., Mandolini, M., Brunzini, A., Gracco, A.: Strength distribution on TMJ using mandibular advancement device for OSAS treatment: a finite element study. Dent. Cadmos 86(08), 757 (2018). https://doi.org/10.19256/d.cadmos. 09.2018 .04

9. Sakhavalkar, P.U., Bhoosreddy, A.R., Kotwal, H.J.: Assessment and comparison of the capsular width of temporomandibular joint on ultrasonography and magnetic resonance imaging. J. Ind. Acad. Oral Med. Radiol. 28, 351-357 (2016). https://doi.org/10.4103/jiaomr.JIAOMR_ 15_16

10. Savoldelli, C., Bouchard, P.O., Manière-Ezvan, A., Bettega, G., Tillier, Y.: Comparison of stress distribution in the temporomandibular joint during jaw closing before and after symphyseal distraction: a finite element study. Int. J. Oral Maxillofac. Surg. 41, 1474-1482 (2012). https://doi.org/10.1016/j.ijom.2012.06.005

11. Wu, J., Liu, Y., Peng, W., Dong, H., Zhang, J.: A biomechanical case study on the optimal orthodontic force on the maxillary canine tooth based on finite element analysis. J. Zhejiang Univ. Sci. B (Biomed. Biotechnol.) 19(7), 535-546 (2018). https://doi.org/10.1631/jzus.B17 00195

12. Liu, Z., Qian, Y., Zhang, Y., Fan, Y.: Effects of several temporomandibular disorders on the stress distributions of temporomandibular joint: a finite element analysis. Comput. Methods Biomech. Biomed. Eng. 19(2), 137-143 (2016). https://doi.org/10.1080/10255842.2014. 996876 
Open Access This chapter is licensed under the terms of the Creative Commons Attribution 4.0 International License (http://creativecommons.org/licenses/by/4.0/), which permits use, sharing, adaptation, distribution and reproduction in any medium or format, as long as you give appropriate credit to the original author(s) and the source, provide a link to the Creative Commons license and indicate if changes were made.

The images or other third party material in this chapter are included in the chapter's Creative Commons license, unless indicated otherwise in a credit line to the material. If material is not included in the chapter's Creative Commons license and your intended use is not permitted by statutory regulation or exceeds the permitted use, you will need to obtain permission directly from the copyright holder.

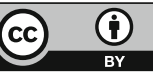

